\title{
ЭКОНОМИКО-МАТЕМАТИЧЕСКОЕ МОДЕЛИРОВАНИЕ
}

УДК $330.46+338.2$

\author{
Д. М.Назаров
}

\section{МОДЕЛЬ РЕФЛЕКСИВНОГО ОТБОРА ИМПЛИЦИТНЫХ ПОКАЗАТЕЛЕЙ УПРАВЛЕНЧЕСКОЙ ДЕЯТЕЛЬНОСТИ ОРГАНИЗАЦИИ}

Статья посвящена описанию экономико-математической модели рефлексивного отбора имплицитных показателей управленческой деятельности организации в рамках классического подхода, реализованного Р. Капланом и Д. Нортоном. В соответствии с построенной моделью вся система сбалансированных показателей деятельности организацией представляется в виде каузального поля, структура которого содержит три подмодели: «имплицитные показатели», «опосредованные показатели» и «ключевые показатели». Такая формализация позволяет не только найти имплицитные показатели деятельности организации при реализации стратегических целей, но и оптимизировать общее количество показателей стратегической карты, оценить силу связей показателей между подмоделями и внутри них на основе теории нечетких множеств, обосновать и согласовать их количественное изменение. Автор приводит пример применения настоящего подхода на основе реально разработанной стратегической карты ООО «ИТЕРУС» и описывает информационную технологию, которая позволяет автоматизировать процесс применения модели, реализованной в виде web-сервиса «Поиск имплицитных параметров». Библиогр. 19 назв. Ил. 4. Табл. 2.

Ключевые слова: рефлексивное управление, система сбалансированных показателей, организация, нечеткие множества, нечеткие бинарные отношения, имплицитные факторы, имплицитные показатели, управленческая деятельность.

\section{M. Nazarov}

\section{MODEL OF REFLEXIVE SELECTION OF IMPLICIT INDICATORS OF MANAGEMENT ACTIVITIES OF AN ORGANIZATION}

The article describes the economic-mathematical model of reflexive selection of implicit indicators of management activities of the organization in the framework of the classical approach implemented by R. Kaplan and D. Norton. According to the constructed model of the whole system of balanced performance indicators of the organization is represented in the form of the causal field, the structure of which contains three submodels: "implicit indicators", "indirect indicators" "key performance indicators". This representation allows not only to find the implicit performance of the organization in implementation of strategic goals, but to balance the total number of indicators, strategic maps, to

Дмитрий Михайлович НАЗАРОВ - кандидат экономических наук, доцент, заведующий кафедрой бизнес-информатики Уральского государственного экономического университета, Российская Федерация, 620000, г. Екатеринбург, ул. 8 Марта, д. 62, каб.450; slup2005@mail.ru

Dmitry M.NAZAROV - PhD of Economics, Associate Professor, Head of the Chair of Business informatics at Ural State Economic University, Russian Federation, 620000, Ekaterinburg, 8 Marta, 62-450; slup2005@mail.ru

(C) Санкт-Петербургский государственный университет, 2017 
estimate the strength of relations between indicators of submodels and within on the basis of fuzzy set theory, to justify and agree on their quantitative changes. The author gives an example application of the described approach based on the developed strategy map, ITERUS, Ltd and describes an information technology to automate the process of applying the model, presented in the form of a web service "Finding implicit parameters". Refs 19. Figs 4. Tables 2.

Keywords: reflexive management, balanced scorecard, organization, fuzzy sets, fuzzy binary relations, implicit factors implicit indicators of management activities.

\section{Введение}

В современных условиях достижение стратегических целей компании осуществляется в рамках построения систем управления корпоративной эффективностью (CPM), фокус которых сосредоточен на выработке решений на основе декомпозиции стратегической цели в виде дерева решения и определения показателей эффективности деятельности компаний. Модели систем сбалансированных показателей (ССП) организации являются важнейшей подсистемой достижения стратегических целей компании в рамках СРМ, поскольку позволяют не только формализовать процесс их достижения, но и организовать мониторинг этого процесса с целью принятия оперативных и тактических решений. Методология ССП, разработанная Р. Капланом и Д. Нортоном [2005], позволяет комплексно формализовать управленческие решения по достижению цели в двух взаимосвязанных плоскостях: 1) выбрать показатели достижения целей и 2) реализовать логику причинно-следственных связей в дереве целей. Основным инструментом реализации этой методологии является стратегическая карта, преимущественное назначение которой - визуализация стратегии по достижению целей развития организации и их мониторинг.

В рамках информационной экономики применение самой методологии ССП является необходимым условием, достаточным же условием с точки зрения ее масштабируемости выступает наличие инструментальных средств, внедренных в практику деятельности хозяйствующих субъектов. Более чем 15-летняя эволюция концепции целевого управления на основе сбалансированной системы показателей деятельности организации позволяет утверждать, что, во-первых, ее используют практически все крупные консалтинговые компании, такие как Accenture, Ernst\&Young, Price Water hause Coopers, KPMG и др.; во-вторых, именно благодаря их накопленному практическому опыту концепция была доведена до «коробочного» продукта и реализована в виде информационной технологии, которая входит во все базовые корпоративные информационные системы (КИС). В частности, все крупные вендоры, такие как Intersoft Lab, BITAM, Business Objects, Cognos, Cristal Decisions, SAS, Hyperion, Pilotsoftware, People Soft, SAP Strategic Enterprise Management, ARISBSC, Oracle BSC и др., опционально в своих КИС имеют инструментальную поддержку концепции ССП [Галахов, 2013].

Разработка и применение той или иной сбалансированной системы показателей конкретной организации целиком зависит от лиц, принимающих решения (ЛПР), а также от наличия инструментальных средств, позволяющих проводить множественные итерации в процессе ее реализации на практике. Практически все эти инструментальные средства носят лишь визуальный характер, позволяют осуществлять мониторинг деятельности организации в режиме on-line, администрируют и регламентируют управленческую деятельность. Как правило, в такого рода 
программных продуктах отсутствует интеллектуальная составляющая, которая могла бы помочь ЛПР избежать ошибок в управлении организацией за счет минимизации «поведенческих паттернов». Термин «поведенческий паттерн» возник в экономической науке благодаря усилению концепции поведенческой парадигмы (роли человека в управлении экономическими агентами рынка), объясняющей ограниченную рациональность (теорию «гарантированного результата» - классику теории игр) в моделях управления экономических систем и их элементов.

Попытку объяснения подобного поведения ЛПР предпринял В. А. Лефевр [Лефевр, Смолян, 1968; Лефевр, 2009]. Он ввел в научный оборот термин «рефлексия» и разработал математическую модель «алгебра конфликта», в основу которой положил формальную математическую логику и теорию графов, рассматривая проблемный контент явления с психологической точки зрения. Целью настоящей статьи является реализация процедуры рефлексивного отбора с применением аппарата теории нечетких множеств, позволяющей выявить имплицитные факторы в системе сбалансированных показателей управленческой деятельности организации и реализовать интеллектуальную поддержку принятия решений. Имплицитный фактор - это неявный, нетривиальный фактор, оказывающий значимое влияние (непосредственно или опосредованно) на деятельность организации, в основе которого лежит скрытая, не учитываемая ранее информация, практически полезная и доступная для генерации знаний в рамках перехода от неформальных к формальным институтам и принятия экономически обоснованных решений. Для достижения поставленной цели были сформулированы следующие задачи: проанализировать предпосылки теории рефлексивного управления организацией; определить роль и место имплицитных факторов в управлении организацией в рамках подхода ССП; построить экономико-математическую модель рефлексивного отбора показателей деятельности организацией; проверить результаты работы на примере построения стратегической карты ООО «ИТЕРУС».

Применение теории рефлексивных игр Лефевра [2009] в рамках построения системы сбалансированных показателей управления деятельностью организации позволяет сформулировать следующую гипотезу: для формирования стратегических целей деятельности организации недостаточно определить высшие ценности ее развития и критерии их достижения и создать стратегическую карту: требуется более тонкая оценка влияния имплицитных факторов, составляющих каузальное поле сбалансированной системы показателей деятельности организации, согласованная со всеми участниками разработки стратегии организации, проведенная в рамках рефлексивного отбора. При этом, учитывая сложность процедуры рефлексивного отбора, необходимо ее автоматизировать и интеллектуализировать с целью сбора, накопления и обработки информации и ее оценки менеджментом организации. В качестве основного модельного инструмента была выбрана теория нечетких множеств, в частности ее раздел нечеткие бинарные отношения и соответствия, позволяющие выявить имплицитные факторы и влияния.

\section{1. Предпосылки применения теории рефлексивного управления в экономике}

Исследования нобелевских лауреатов убедительно показали, что постулаты классической экономической теории во многом претерпевают изменения с тече- 
нием времени и накоплением практического опыта, смещаясьв сторону поведенческой парадигмы.

В 1993 г. нобелевская премия была вручена Д. Норту и Р. Фогелю за два взаимодополняющих открытия в области экономики. Норт переосмыслил понятия институтов, ассоциировав их с «правилами игры», и провел исследование проблем изменения самих институтов с точки зрения устойчивости неэффективных норм и правил [North, 1991].Фактически он декомпозировал их на две взаимосвязанные структуры - формальные и неформальные институты. Главной заслугой Р. Фогеля и его коллег - основателей «клиометрии» является радикальное обновление методов историко-экономических исследований на базе достижений эконометрики, статистического и экономического анализа[Fogel, 1964]. Он впервые обратил внимание на то, что несущественные нововведения в промышленности в большей степени способствуют ее эволюции, чем крупные технологические открытия.

Начиная с 2001 г. премии были вручены за исследования, которые можно условно объединить под названиями: «Информационная экономика» (Дж. Акерлоф, М.Спенс, Дж. Ю.Стиглиц); «Психологическая и экспериментальная экономика» (Д.Канеман, В.Л.Смит); «Коммуникативная экономика» (И. Р.Дж. Ауманн, Т.К.Шеллинг); «Экономические механизмы» (Л. Гурвич, Э. Маскин, Р. Б. Майерсон, П.Кругман, Э.Остром, О. Уильямсон, П.Даймонд, Д. Мортенсен, К.Писсаридес). В работах [Stigler, 1965; Kahneman, Tversky, 1979; Рязанов, 1998; Нейман, Моргенштерн, 1970; Канке, 2007] развиваются проблемы, затронутые нобелевскими лауреатами, эти исследования вносят значительный вклад в классическую экономическую теорию, наполняя уже существующие и проверенные положения новым содержанием, в частности описывая с разных позиций вопросы обеспечения оптимального поведения экономических агентов. Принцип неравномерного распределения информации («информационная экономика») способствовал возникновению идеи ограниченной рациональности в поведении участников рынка. Модели принятия решений в условиях неопределенности с целью оптимизации поведения агента рынка («психологическая и экспериментальная экономика») привели к осознанию того, что экономика «человекоразмерна» и этот факт необходимо учитывать при описании поведения агентов рынка. Расширенная интерпретация проблемы конфликта и кооперации в рамках теории игр обусловило введение в научный оборот понятия «фокальные точки». Они характеризуют состояния наиболее вероятного равновесия, предложенные ЛПР, которые не всегда совпадали с результатами расчетов в рамках классической теории игр («коммуникативная экономика»). Модели, реализованные в рамках теоретико-методологических исследований различных по своей природе экономических механизмов, подтвердили более ранние исследования и позволили более качественно объяснить возникающие в практической деятельности проблемы и парадоксы.

Одним из первых объяснить поведение человека в процессе принятия решений с психологической точки зрения попытался В.А. Лефевр, обосновав теорию рефлексивного управления. Он расширил границы понятия «рефлексия», выделив рефлексию первого (авторефлексию) и второго рода. Смысл авторефлексии - отражение, исследование познавательного акта в преломлении к своим действиям, к своим мыслям; рефлексия второго рода заключается в принятии решений субъектом на основе авторефлексии других субъектов. Такое толкование изучаемого 
явления породило возникновение нового термина - «рефлексивное управление», который первоначально использовался в военной сфере, - в частности, «рефлексивное управление» интерпретировалось как «процесс передачи оснований для принятия решения одним из противников другому». В качестве оснований могли использоваться любые обманные движения, провокации и интриги, маскировки, розыгрыши, создание ложных объектов (и вообще ложь в любом контексте) [Лефевр, 2009].

Таким образом, рефлексивное управление - это методология управления, в рамках которой необходимо предвидеть поведение системы как дуального взаимодействия «объект-субъект» в условиях изменяющейся окружающей среды. Иными словами, это процесс «рефлексивного программирования» партнера или противника посредством специально подготовленной информации с целью склонить его добровольно принять предопределенное решение, желательное для инициатора действия.

Вопросы рефлексивного управления в экономике были глубоко проанализированы и описаны в трудах украинских ученых под руководством Р. Н. Лепа [Лепа, Мальчик, 2010], который рассматривает поведение ЛПР в условиях ограничения времени и биполярного выбора «принять или не принять» и утверждает, что если с точки зрения ЛПР вопрос не настолько важен, то решение будет принято по «образцу», с опорой на интуицию и знания, с поиском аналогов во внешней среде, без глубокого анализа имеющихся данных, привлечения экспертов и т. д. То есть имеется применение двух типов рефлексий: первого рода (собственный опыт, интуиция) и второго рода (с опорой на внешний опыт ЛПР в схожих ситуациях).

Такое управление неизбежно приведет к ошибкам, и чтобы их минимизировать, необходимо разрабатывать модели экономических систем, которые, с одной стороны, учитывали бы их рациональное поведение, апеллируя к уже хорошо известным науке и практике показателям; с другой - нивелировали бы рефлексивные воздействия за счет интеллектуализации деятельности ЛПР, минимизирующей рутинную работу при выборе альтернатив.

Рефлексивное управление осуществляется на основе моделей Лефевра, которые условно можно определить как модель «успех-неуспех», модель «союз-конфликт», модель биполярного выбора, модели рефлексивных игр.

В модели «успех-неуспех» возникает многоярусный каскад генерации высших ценностей, которые предопределяют выборы субъектов в зависимости от качества отношений между ними: успех одного контрагента рынка металлов может как усилить, так и уменьшить ощущение успеха у другого [Лепа, Мальчик, 2010; Лепский, 2014].

В модели «союз-конфликт» определяющее значение имеет отношение одного контрагента рынка к другому. Выбрать это определенное состояние отношений можно только с помощью системы высших ценностей. В модели учитывается важная связь между этической системой лиц, принимающих решения, и выбором отношений к другому субъекту. В первой этической системе, где соединение позитивных (1) и негативных тенденций (0) оценивается как негатив (0), нормативно один субъект стремится вступить в кооперацию с другим субъектом (1); во второй этической системе он склонен к конфронтации (0). Эмпирический анализ показал, что в модели нашли отражение существенные различия нормативных оценок от- 
ношения одного контрагента рынка к другому в различных культурах [Лефевр, 2009; Лепский, 2014].

В модели биполярного выбора подчеркивается, что экономическое поведение субъекта неоднозначно и зависит от некоторого ключа, критерия, который служил бы «переключателем» между позитивом и негативом [Лефевр, 2009; Лепский, 2014]. Другими словами, оно может существенно отклоняться от оптимального гарантированного в парадигме результата.

В теории рефлексивных игр общей ценностью является принцип запрета эгоизма: каждый субъект, входящий в группу, преследуя свои личные цели, не должен наносить ущерб группе как целому, т.е. действия, выгодные для субъекта, но не выгодные для группы, являются неприемлемыми [Лефевр, 2009; Лепский, 2014]. При этом подобные действия принципиально не запрещены, если лицо, их совершающее, не получает никакой выгоды. Принцип запрета эгоизма имеет в теории рефлексивных игр примерно то же значение, что и принцип гарантированного результата в классической теории игр. Теория рефлексивных игр позволяет предсказывать выборы субъектов, входящих в группу, если известен граф отношений между субъектами и их воздействия друг на друга.

Актуальность изучения проблем рефлексивного управления подтверждается работами других известных ученых. В частности, нобелевский лауреат Р. Ауманн в своих исследованиях ввел понятие «общее знание» в рамках рефлексии объектов экономической системы, которое определяло ценностные ориентации их поведения.

Таким образом, на каждом «витке спирали» модели управления хозяйственной деятельностью организации важно применять принципы рефлексивного управления, основанные на соответствующих моделях с целью минимизации «битых» паттернов поведения ЛПР. Для этого на основе интеллектуального анализа данных и их интерпретации ЛПР должно проводить реблексивный отбор показателей эффективности деятельности предприятия в рамках лефевровской модели рефлексивных игр и биполярного выбора («ложь» или «истина»). На наш взгляд, к процессам рефлексивного отбора целесообразно применять математический аппарат теории нечетких множеств. Присутствие человека (ЛПР, персонал и т.д.) всегда вносит в производственный и управленческий процесс неопределенность, которую современный математический аппарат может учитывать лишь частично. Законы справедливые и статистически значимые для технических систем при воздействии на них человеческого фактора - неотъемлемой части любого производства - трансформируются в закономерности, которые, в отличие от строгих математических формул, описывающих законы, способны в ряде случаев показать исключительно качественные характеристики процесса или явления. Но постановка задачи в данной формулировке в рамках перехода к очередному технологическому укладу становится слишком узкой, поскольку на экономическую деятельность оказывают влияние имплицитные факторы, при этом, во-первых, в традиционных моделях управления организации по показателям они не учитываются; во-вторых, с помощью традиционных моделей формальной логики и теории графов по Лефевру не представляется возможным их выявить, поскольку размываются понятия «истина» и «ложь». 


\section{2. Рефлексивное управление в системе сбалансированных показателей деятельности организации с учетом имплицитных факторов}

Сбалансированная система показателей концептуально входит в класс информационных систем под названием Business Performance Management (BPM). Это не только новая управленческая концепция, но и один из наиболее быстро растущих секторов IT-решений. ВРМ включает набор методологий и инструментальных средств, которые помогают эффективно планировать, измерять, анализировать и повышать эффективность бизнес-процессов организации.

ВРМ-системы покрывают разрыв функциональности Decision Support System, DSS (системы поддержки принятия решений) и Business intelligence, BI (системы бизнес-аналитики), позволяя автоматизировать корпоративное моделирование бизнес-процессов, обеспечивают мониторинг и контроль ключевых показателей деятельности организации, являются эффективным инструментом как стратегического, так и оперативного планирования.

Система сбалансированных показателей деятельности становится основой управления организацией на определенном этапе ее стратегического развития, поэтому имеет смысл говорить о том, что ВРМ-система является информационной реализацией процессной модели хозяйственной деятельности предприятия.

Процессная модель управления, во-первых, позволяет представить внутреннюю среду организационно-производственной системы в качестве совокупности бизнес-процессов, которая описывается системой сбалансированных показателей эффективности деятельности, декомпозируемой применительно к отдельному бизнес-процессу; во-вторых, отражает трансформацию любого сигнала или ресурса, поступившего из внешней среды, в некую ценность - результат деятельности предприятия; в-третьих, реализуясь в условиях информационной экономики, накладывает особый отпечаток на эффективное использование информационных ресурсов.

Развитие в рамках такой модели идет по «спирали», создавая при прохождении нового витка очередной функциональный модуль, при этом стадии, уровни и типы бизнес-процессов не разделены однозначно по фазам и могут выполняться параллельно, большинство их них имеет сквозной характер, т. е. они выполняются сразу в нескольких фазах. Результатом деятельности на определенном витке «спирали» является переосмысление процесса, выбор новой или уточнение старой модели эффективности деятельности, а также интерпретация результатов деятельности и накопление знаний и опыта в рамках конкретного предприятия, его обобщение и распространение.

Центральным звеном процессной модели управления хозяйственной деятельностью являются интеллектуальные способности и системное мышление ЛПР, а также возможности информационных систем бизнес-аналитики, способных взять на себя рутинную задачу по преобразованию данных в информацию и знания, которые выступают достаточным условием применения методологии на практике.

На выработку стратегической цели, а впоследствии и стратегического решения влияет ряд факторов: глобальные и локальные тренды экономического развития в целом и отраслевого развития в частности, видение ситуации акционерами и другими заинтересованными лицами, менеджментом компании на основе нако- 
пленных знаний и практического опыта, а также лучшие международные практики и мнения экспертных сообществ разного уровня.

В явном виде критика существующей модели ССП приводится в работе А.О.Недосекина и соавторов, которые отмечают, что в традиционной модели ССП, построенной по типу модели Нортона-Каплана, во-первых, не учитываются интересы всех стейкхолдеров, которые формируют баланс рыночных отношений организации в экономической системе; во-вторых, нет инструментов работы с качественными и признаковыми факторами; в-третьих, не берутся во внимание связи между проекциями, игнорирование которых в большинстве случаев приводит к ошибкам управления. Авторы предлагают формализацию модели ССП в виде нечетко-логического графа (это позволит учесть обратные связи в системе показателей, а также выявить наличие петель) и приводят пример калибровки связей ССП на основе теории нечетких множеств, используя стандартный инструментарий пяти- или трехмерного нечеткого классификатора [Недосекин и др., 2013].

В условиях информационной экономики, когда потоки информации становятся критическими в системе управления деятельностью объекта управления, проявляется влияние имплицитных факторов, которое в традиционной модели ССП не учитывается, но при этом может оказывать существенное воздействие на результаты деятельности организации. Заметим, что природа этих факторов требует дополнительного исследования в части классификации, анализа уровней и оценки результатов влияний каждого из них. Имплицитные факторы в управлении становятся заметными вследствие влияния очевидных факторов, уже учитываемых в системе при неэффективном управлении. Приближение обозначенной трактовки к терминам моделей рефлексии позволяет утверждать, что источником их возникновения могут служить противоречия и конфликты системы управления с интересами управляемого объекта. Это приводит к динамической неустойчивости системы управления и, как правило, способствует возникновению временных объединений работников организации, которые преследуют различные интересы и по-разному реагируют на достижение стратегических, тактических и оперативных целей. Таким образом, классическая теория ССП дает возможность построить каркас измеряемых показателей управленческой деятельности, но не определяет интеллектуальные инструменты для построения связей между ними, более того, в ней не учитывается характер таких связей.

Механизмы влияния имплицитных факторов на результаты деятельности организации изучены недостаточно, последствия оказываемого влияния можно продемонстрировать на основе положений синергетики.

До тех пор пока организация, рассматриваемая как система, движется в направлении аттрактора, предельного положения состояния, ее поведение поддается прогнозированию. Вблизи предельных состояний, т. е. при достижении экстремальных результатов хозяйственной деятельности, возникает область неустойчивых состояний - точки бифуркации, в которых наблюдается эффект бабочки (малое воздействие внешних сил может привести систему к диаметрально противоположным векторам развития: к краху или более высокому уровню развития). В этом заключается принцип резонанса [Назаров, 2012], применение которого к бизнеспроцессам, протекающим в организации, предполагает учет неявно содержащихся в системе сбалансированных показателей деятельности имплицитных факторов, 
которые, во-первых, оказывают существенное влияние на стратегические результаты деятельности организации; во-вторых, обусловливают вектор ее развития; в-третьих, корректируют ценностные ориентиры ее деятельности.

С учетом принципа резонанса система сбалансированных показателей деятельности предприятия должна быть модифицирована путем включения в ее модель имплицитных факторов, которые позволяют производить комплексный учет различных видов неопределенности, связанных с неожиданными потерями, убытками, негативными сценариями развития событий. В такую систему должны быть заложены риски производственной деятельности, риски планирования - как стратегического, так и оперативного, риски маркетинговых исследований, финансовые риски.

\section{3. Модифицированная система сбалансированных показателей деятельности организации}

Опишем модифицированную ССП с учетом введения в нее имплицитных факторов (рис. 1). Не нарушая положений концепции Р. Нортона и Д. Каплана в целом, предложим формализовать имплицитный фактор в виде совокупности измеримых показателей, однозначно идентифицирующей его в рамках системы ценностных ориентиров, принятых в конкретной организации. При этом обеспечивается возможность управления имплицитным фактором через изменение управляющих воздействий на его показатели.

Следующий концептуально важный элемент ССП - причинно-следственные связи, формирующие логику достижения стратегической цели, которая должна быть модифицирована с учетом влияния имплицитных факторов. Принимая необязательное наличие имплицитных факторов в каждой из проекций, необходимо предложить структурную модель, позволяющую выявить имплицитные влияния и учесть их в процессе управления. В результате устанавливается каузальность связей показателей достижения цели и имплицитных факторов.

Для проектирования модели учета имплицитных влияний сформулируем гипотезу о том, что имплицитные факторы воздействуют на основные показатели деятельности организации опосредованно. Это означает, что все показатели, которые выбраны для реализации стратегии развития организации в рамках ССП, необходимо декомпозировать на 3 составляющие: подмодель А характеризует имплицитные факторы в виде системы показателей, подмодель В представляет собой совокупность опосредованных показателей, а подмодель C - множество основных показателей деятельности организации, которые, как правило, способствуют увеличению рыночной и акционерной стоимости организации.

Таким образом, необходимо провести отбор соответствующих показателей и построить между ними новую логику, реализующую причинно-следственные (каузальные) связи. В настоящей работе мы предлагаем реализовать этот отбор с использованием моделей рефлексивного управления. Это объясняется тем, что набор показателей ССП не статичен: его динамика определяется в соответствии с принципами рефлексивного управления, а также интеллектуальным анализом хозяйственной и управленческой деятельности организации (рис. 2). 


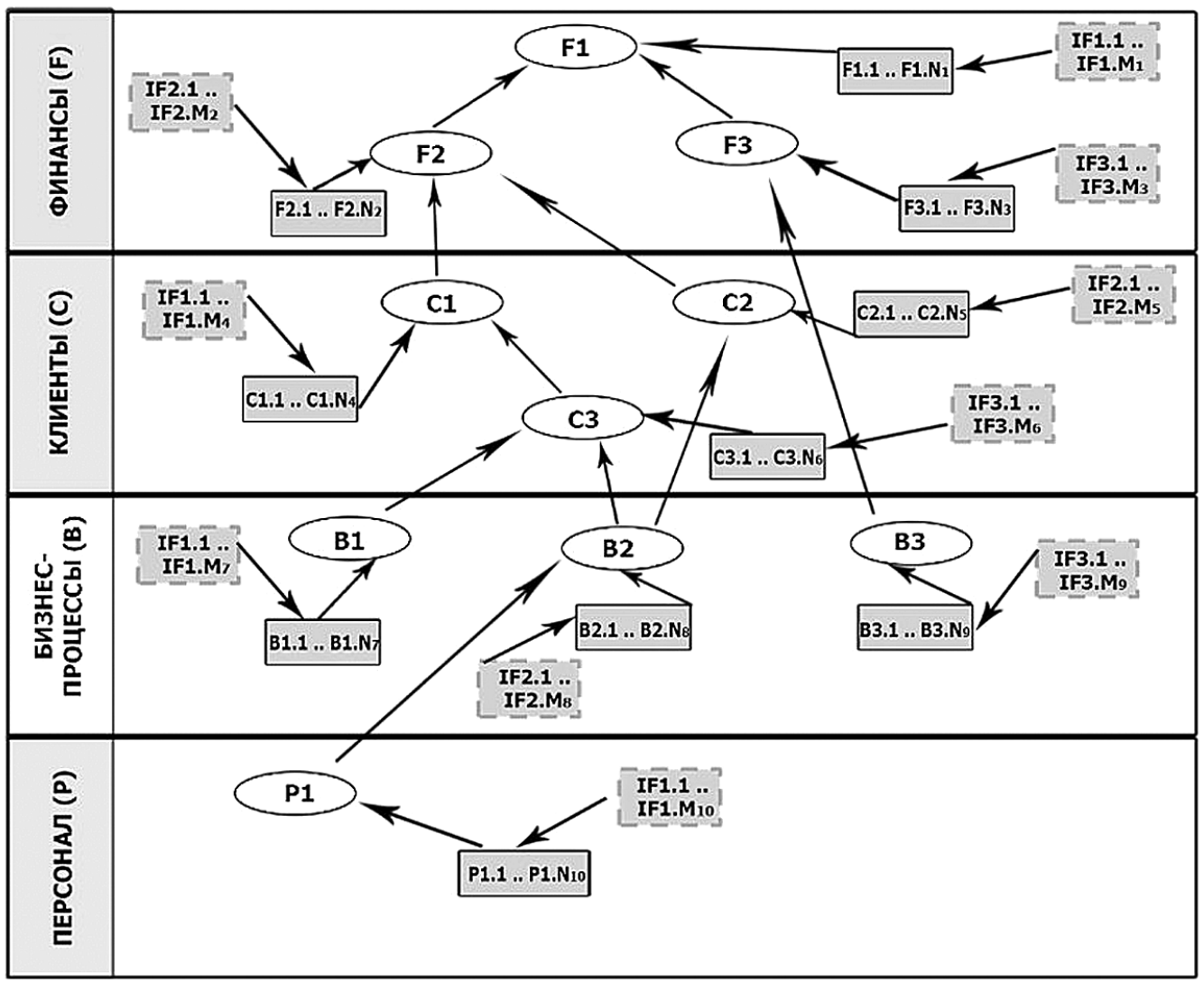

Puc. 1. Модифицированная стратегическая карта модели ССП

О 6 о з н ач ен и я : $F 1-$ стратегические целевые установки; $F 1.1-F 1 . N_{1}-$ показатели, по которым осуществляется мониторинг достижения цели $F 1$; IF1.1- IF1.M - показатели имплицитных факторов, оказывающих влияние на показатели достижения стратегических целей.

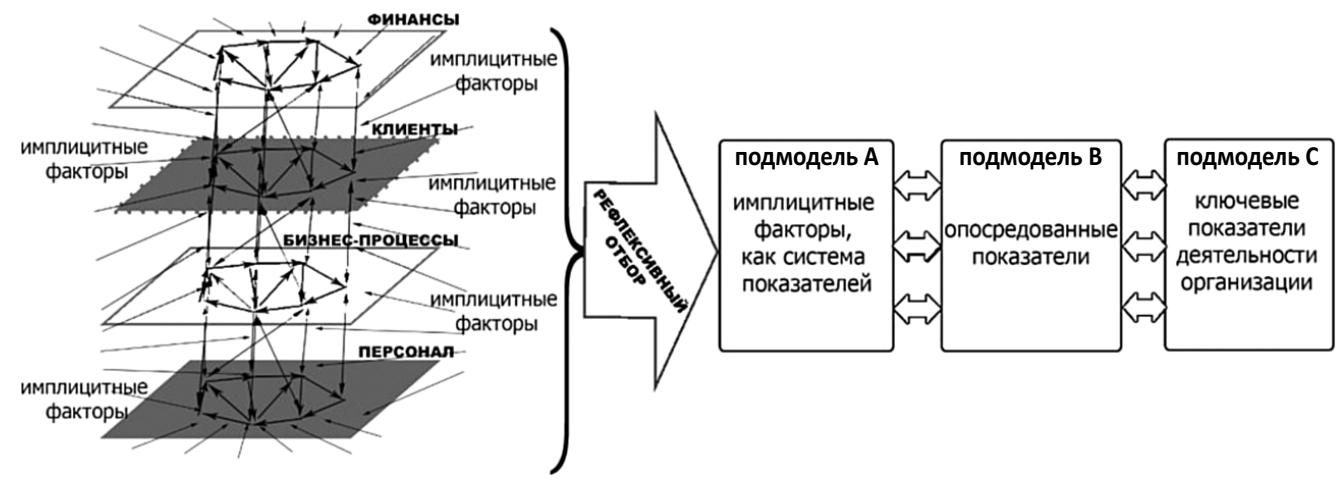

Puc. 2. Модель рефлексивного отбора для оценки влияния показателей имплицитных факторов на ключевые показатели деятельности организации 
На рис. 2 представлена рамочная технология рефлексивного отбора показателей деятельности хозяйствующего субъекта, которая позволяет провести оценку влияния имплицитных факторов на основные показатели его деятельности. Модель является дополнением традиционной модели ССП, поскольку требует реализации еще одной процедуры - рефлексивного отбора и построения на его основе подмоделей А, В, С.

В рамках процедуры рефлексивного отбора ЛПР ставит каждому показателю в соответствие значение «0» или «1» на основе логики: «не включать показатель» или «включать показатель» в подмодель. В настоящей статье мы предлагаем дополнение модели Лефевра, которая на основе теории нечетких множеств с использованием лингвистических описаний принимаемого решения ЛПР позволит выбрать оптимальную систему показателей с учетом наличия в ней имплицитных факторов.

В такой постановке задача оценки имплицитных факторов и их влияния может быть решена с помощью технологий нечеткого управления.

\section{4. Модель выявления имплицитных факторов в системе сбалансированных показателей деятельности}

На современном этапе развития экономико-математических методов в качестве альтернативы традиционным подходам возможно использовать модель нечеткого управления, которая позволяет эффективно учитывать неопределенность, неточность, неполноту, неколичественность и имплицитность.

Большая часть информации, необходимой для описания основных бизнеспроцессов, существует в форме представлений или намерений ЛПР либо экспертов и формализуется в рамках корпоративных информационных систем. Главная трудность в использовании такой информации заключается в сложности учета влияния представлений на основной бизнес-процесс и, как следствие, на результаты принятия решения по той или иной проблеме.

При математическом моделировании сложной системы (процесса) важно не только принять во внимание исчерпывающий перечень реальных факторов, оказывающих влияние на результат, но и показать степень их воздействия в разных условиях. Нечетко-множественный подход позволяет при этом учесть каузальность на первый взгляд несущественных имплицитных факторов, используя слова естественного языка «сильно», «слабо» и т. д. Операции такого типа основаны на допущении, что отклик модели на воздействие может быть известным лишь приближенно, нечетко.

Задачей построения модели оценки каузальности является обнаружение и вычисление влияния имплицитных факторов на связанные непосредственно с ними показатели и через них - на основные показатели экономической деятельности предприятия. При этом существенной частью построенной модели будет выявление самих имплицитных факторов в рамках протекающих бизнес-процессов и трудно формализуемых социально-общественных явлений.

Основой для построения модели послужит технология построения нечетких бинарных отношений через их композицию.

Пусть задано некоторое непустое конечное множество $A=\left\{a_{1}, a_{2}, \ldots, a_{n}\right\}$. Как правило, эксперты вполне четко могут определить степень влияния элементов 
множества друг на друга, однако выявление опосредованных влияний - достаточно сложная задача. Так, возможна ситуация, при которой какие-либо два элемента множества практически не влияют друг на друга, но существует элемент, отличный от этих двух, через который это влияние становится существенным. Такое опосредованное влияние нельзя не учесть, поскольку это приведет к неадекватным результатам использования модели.

Выявление опосредованных влияний основано на технологии оценки нечетких бинарных отношений на некотором множестве [Назаров, Конышева, 2011].

Введем некоторые определения.

Пусть $U-$ непустое конечное множество, $U^{2}$ - декартов квадрат этого множества $\left(U^{2}=U \times U=\{(a ; b): a, b U\}\right)$.

Нечетким бинарным отношением на множестве $U$ называют нечеткое подмножество $U^{2}$ :

для дискретного множества

$$
\Gamma=\sum_{U^{2}} \mu_{\Gamma}\left(u_{i}, u_{j}\right) /\left(u_{i}, u_{j}\right),
$$

для непрерывного множества

$$
\Gamma=\int_{U^{2}} \mu_{\Gamma}(x, y) /(x, y) .
$$

Функция принадлежности нечеткого бинарного отношения $\mu_{\Gamma}(x, y)$ является аналогом характеристической функции в случае обычных бинарных отношений. При этом каждому нечеткому бинарному отношению можно поставить в соответствие матрицу, элементами которой будут значения функции принадлежности. Такие матрицы в теории нечетких множеств принято обозначать $J_{\Gamma}$.

Композииией нечетких бинарных отночений $\Gamma_{1}$ и $\Gamma_{2}$ называют нечеткое бинарное отношение $\Gamma=\Gamma_{1} \circ \Gamma_{2}$, причем

$$
\mu_{\Gamma_{1} \circ \Gamma_{2}}(x, y) /(x, y)=\bigcup_{z \in U}\left(\left(\mu_{\Gamma_{1}}(x, z) /(x, z)\right) \bigcap\left(\mu_{\Gamma_{2}}(z, y) /(z, y)\right) .\right.
$$

Пересечение одноточечных нечетких множеств $\mu_{\Gamma_{1}}(x, z) /(x, z)$ и $\mu_{\Gamma_{2}}(z, y) /(z, y)$ обычно выполняется по логической $T$-норме, а объединение по логической T-конорме: $a \cap b=\min (a, b), a \cup b=\max (a, b)$.

При этом формула (3) принимает вид

$$
\mu_{\Gamma_{1} \circ \Gamma_{2}}(x, y) /(x, y)=\max _{z \in U}\left(\min \left(\mu_{\Gamma_{1}}(x, z), \mu_{\Gamma_{2}}(z, y)\right) /(x, y) .\right.
$$

График композиции отношений определяется следующими формулами: для дискретного случая

$$
\Gamma_{1} \circ \Gamma_{2}=\sum_{U^{2}} \mu_{\Gamma_{1} \circ \Gamma_{2}}(x, y) /(x, y)=\sum_{U^{2}}\left(\max \left(\min \mu_{\Gamma_{1}}(x, z), \mu_{\Gamma_{2}}(z, y)\right)\right) /(x, y),
$$

если $U-$ конечное множество; 
для непрерывного случая

$$
\Gamma_{1} \circ \Gamma_{2}=\int_{U^{2}} \mu_{\Gamma_{1} \circ \Gamma_{2}}(x, y) /(x, y)=\int_{U^{2}} \max _{z \in U}\left(\min \mu_{\Gamma_{1}}(x, z), \mu_{\Gamma_{2}}(z, y)\right) /(x, y),
$$

если U часть числовой оси или вся числовая ось.

Из формулы (4) следует, что для случая, когда U - конечное множество, матрица композиции отношений $J_{\Gamma_{1} \circ \Gamma_{2}}$ есть максиминное произведение матриц $J_{\Gamma_{1}}$ и $J_{\Gamma_{2}}:$

$$
J_{\Gamma_{1} \circ \Gamma_{2}}=J_{\Gamma_{1}} \circ J_{\Gamma_{2}}=\left(\operatorname { m a x } _ { k } \left(\min \left(\mu_{\Gamma_{1}}\left(u_{i}, u_{k}\right), \mu_{\Gamma_{2}}\left(u_{k}, u_{j}\right)\right)_{n x n}=\left(\mu_{\Gamma_{1} \circ \Gamma_{2}}\left(u_{i}, u_{j}\right)\right)_{n x n},\right.\right.
$$

где $n$ - число элементов множества $U$.

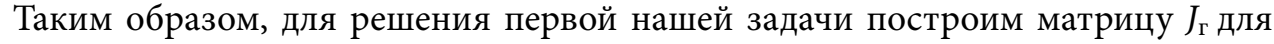
множества А, определенного выше.

$$
J_{\Gamma}=\left(\begin{array}{cccc}
s_{11} & s_{12} & \ldots & s_{1 n} \\
s_{21} & s_{22} & \ldots & s_{2 n} \\
\ldots & \ldots & \ldots & \ldots \\
s_{n 1} & s_{n 2} & \ldots & s_{n n}
\end{array}\right) \text {, где } s_{i j}\left(0 \leq s_{i j} \leq 1 ; \quad i=1,2, \ldots, n ; j=1,2, \ldots, n\right) .
$$

Интерпретируем элементы матрицы $J_{\Gamma}$ как силы влияния показателя $a_{i}$ на показатель $a_{j}$. Значения $s_{i j}$ обычно определяются экспертами с учетом использования именно лингвистических переменных и соответствующих им характеристик: «очень слабо», «слабо», «средне», «сильно», «очень сильно». Для проверки согласованности, уточнения, повышения обоснованности экспертных оценок величин $s_{i j}$ может быть использован экспертный метод, например, метод анализа иерархий и другие, в том числе и наш, описанный выше в настоящей статье.

Используя максиминное умножение элементов матрицы по формуле (4), можно выявить опосредованные влияния внутри элементов множества, т. е. найти значения элементов матрицы $J_{\Gamma^{2}}$ :

$$
J_{\Gamma^{2}}=J_{\Gamma} \cdot J_{\Gamma}=\left(\begin{array}{cccc}
f_{11} & f_{12} & \cdots & f_{1 n} \\
f_{21} & f_{22} & \cdots & f_{2 n} \\
\ldots & \cdots & \cdots & \cdots \\
f_{n 1} & f_{n 2} & \cdots & f_{n n}
\end{array}\right) .
$$

$s_{i j}<<f_{i j}$ указывает на наличие имплицитного влияния, опосредованного каким-либо третьим фактором, присутствующим в системе. Достоинством модели является возможность выявления как опосредованных, так и имплицитных факторов [Назаров, 2016].

На основании изложенной выше математической модели был разработан webсервис, позволивший унифицировать технологию опытно-экспериментальной работы, включая контроль доступа, администрирование, аккумуляцию информации и знаний, создать условия для совместной работы экспертов, специалистов предметных областей и программистов. Разработанный сервис включает в себя основные средства проведения расчетов по моделям оценки имплицитных факторов, прежде всего набор программ, реализующих интеллектуальный анализ входных данных предметной области на основе аккумуляции экспертных мнений с учетом 
их согласованности методом рандомизированных сводных показателей (Aggregate Indices Randomization Method, AIRM). Также сервис содержит набор программных утилит, обеспечивающих взаимодействие с другими подсистемами в режиме реального времени, для которых в рамках стандартных средств и процедур разработаны интерфейсы для трансформации данных в форматы входных данных программ модулей. С программной точки зрения сервис является кроссплатформенным, написан на языке РНР с использованием встроенных в среду программных библиотек и оконного интерфейса. В сервисе имеется подсистема баз данных предметной области, которая содержит структурированную пополняемую реляционную базу знаний о моделируемых объектах и процессах, в частности наборы исследуемых расчетных данных, процедуры расчета, модели и наборы показателей, а также стандарты взаимодействия баз данных и работы с ними.

Web-сервис «Поиск имплицитных факторов» использовался нами для моделирования процессов поиска имплицитных факторов в экономических системах и реализации процедуры рефлексивного отбора. Обратим внимание на то, что разработанная экономико-математическая модель позволяет ЛПР интеллектуализировать процесс выявления имплицитных факторов, а также выявлять сильные и слабые взаимовлияния факторов и показателей управления с учетом наличия в системе имплицитных факторов.

\section{5. Применение модели к разработке модифицированной системы сбалансированных показателей деятельности организации}

Наличие показателей в стратегической карте есть условие необходимое, которое понимается большинством исследователей как инструмент стратегического контроля или мониторинга, но недостаточное, поскольку набор показателей без привязки к установленным механизмам контроля не применим к процессу принятия решений. Важнейший вопрос для менеджмента компаний, исследователей, ученых и практиков заключается в целесообразности включения тех или иных показателей в стратегические карты. Это вызвано тремя главными причинами: 1) показатель не в полной мере отражает особенности того или иного аспекта деятельности; 2) методика расчета показателей субъективна; 3) смысл показателя относительно объекта его применения может изменяться как в статике, так и в динамике. Методика Д. Нортона, Р. Каплана последовательно позволяет увязать цели, показатели и результаты исходя из следующей логики: под показателями понимаются краткие характеристики хозяйственных процессов в количественном или в качественном выражении, хозяйственные процессы отражают определенную сторону деятельности изучаемой компании, а исследуемая сторона деятельности определяется стратегической целью. Обратим внимание на то, что такой подход легко увязывается в триаду «данные - информация - знания». Данные представляют собой результат измерения и, как правило, имеют количественное выражение, однако информацией они становятся в том случае, если адресат знает методику их измерения, понимает ценность и в конечном итоге они уменьшают степень неопределенности. Новое знание при этом возникает лишь тогда, когда информация имеет значение для лица, получающего ее. Иными словами, определение системы показателей формирует тезаурус, с помощью которого исследователи пытаются донести свое 
мнение об эмпирически полученных результатах и изучаемом процессе, а адекватность тезауруса определяет степень соответствия показателей процессу или явлению. Широта сферы использования показателей определяет их системную сложность, понимаемую нами как комплексность в системе их отбора, определения, толкования и в методике измерения, которая создает необходимость тщательного экспертного отбора показателей с учетом пожеланий не только ученых, но и ЛПР. В этом, на наш взгляд, проявляется рефлексивная сущность любой системы показателей, а следовательно, и рефлективность в сфере их мониторинга и интерпретации в рамках проводимого исследования. Количество принимаемых к расчету показателей определяется на основе теоретических разработок, зависит от практической целесообразности и мирового опытасоставления стратегических карт для различных компаний. На корпоративном уровне и уровне отдельных подразделений, согласно практическому руководству по оценке эффективности деятельности компаний, обычно используется 15-25 показателей; на уровне функционального отдела их количество сокращается до 10-15; на уровне участка или отдельного работника требуется 5-10 показателей [Ольве, Нильс-Горан, 2004].

Таким образом, при составлении стратегических карт необходимо стремиться отобрать оптимальную систему показателей, которую получают на основе решения пяти задач:

1) обобщения наблюдений за некоторым количеством компаний в определенной отрасли и сегменте рынка;

2) определения существенных показателей деятельности, которые отражают свойства изучаемого процесса, объекта или явления (они получены в результате периодических наблюдений);

3) выявления потребности ЛПР и выбираемых ими конкретных целей с заранее заданными ориентирами и методиками расчета;

4) получения доступа к новой информации, имеющейся у отдельных сотрудников компании и неизвестной остальным, и создания на их базе нового знания;

5) обеспечения совпадения тезаурусов источников и получателей информации.

При выборе системы показателей в рамках концепции ССП необходимо выстроить логическую цепочку, опираясь на план стратегического развития компании, в соответствии с декомпозицией по четырем перспективам. Развитие следует представлять как взаимосвязанный процесс, требующий новых знаний и компетенций сотрудников (перспектива «обучение и рост»); внедрения новых управленческих и производственно-предметных технологий (перспектива «бизнес-процесс»), что позволит уверенно позиционировать компанию на соответствующем сегменте рынка (перспектива «клиенты») и приведет к желаемому финансовому и экономическому результату (перспектива «финансы»).

Продемонстрируем процесс разработки модифицированной ССП для ООО «ИТЕРУС». Общая структура ССП представлена в табл. 1.

В ходе анализа бизнес-процессов ООО «ИТЕРУС» был выявлен 21 показатель, каждый из которых отражает эффективность исполнения одной из задач или одной из целевых функций. Построенная нами стратегическая карта удовлетворяет 


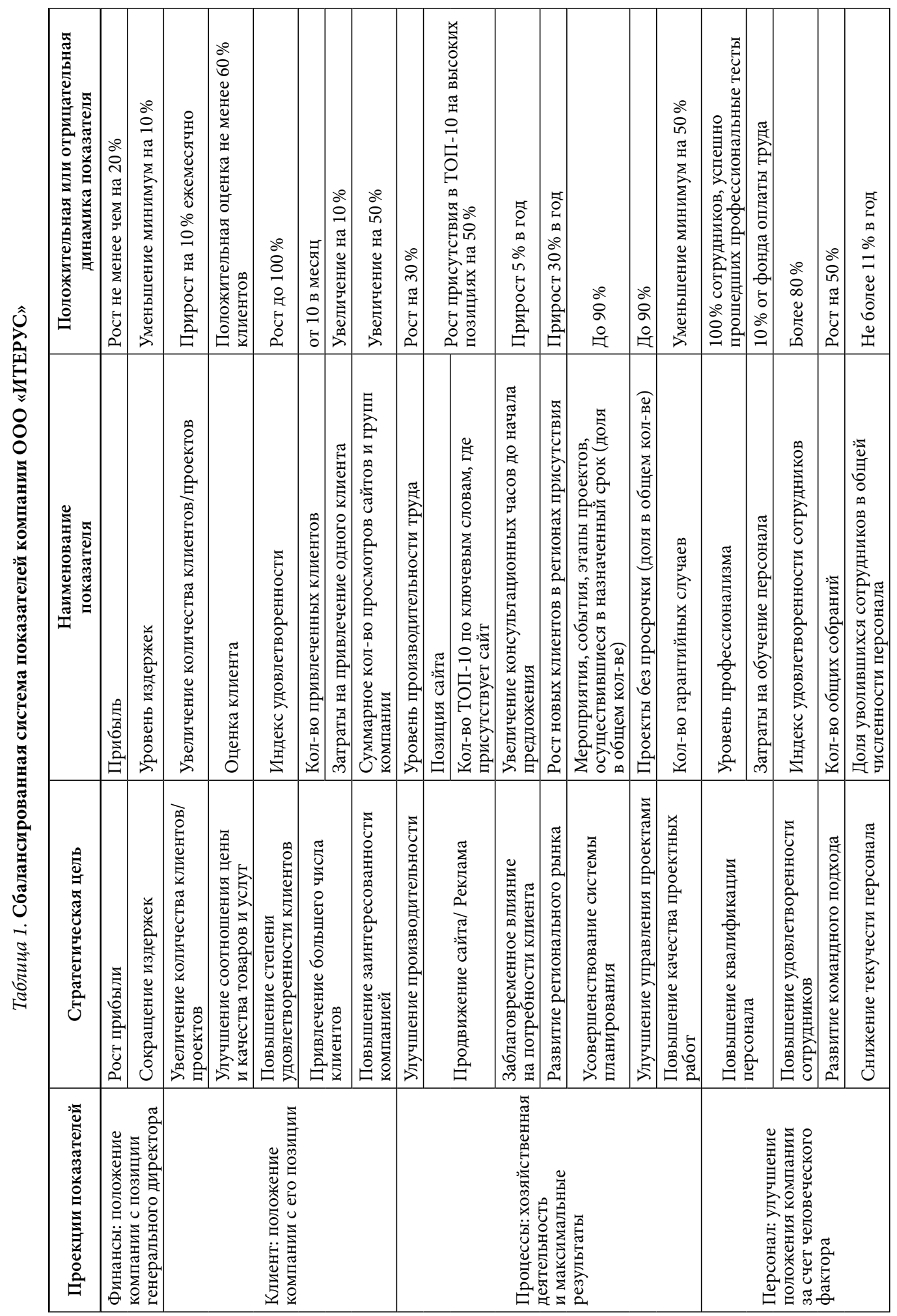


основным требованиям к ее разработке и служит иллюстрацией превращения абстрактных целей в четкие верифицируемые показатели, одинаково понятные и исследователям, и руководителям компаний.

Алгоритм рефлексивного отбора:

Шаг 1. Формирование показателей эффективности деятельности (стратегическая карта)

Шаг 2. Введение этих показателей в систему с помощью web-сервиса «Поиск имплицитных факторов»

Шаг 3. Оценка их взаимного влияния по шкале «очень слабо», «слабо», «средне», «сильно», «очень сильно»

Шаг 4. Проведение интеллектуального анализа введенных данных и оценка полученных результатов.

Шаг 5. Если результат Шага 4 не устраивает ЛПР, то осуществляется переход на Шаг 1.

Для полученной системы показателей, в соответствии с процедурой рефлексивного отбора и разработанного нами инструментального метода ее реализации нахождения имплицитных факторов в рамках ССП, был произведен эксперимент, который заключался в выявлении каузального поля показателей деятельности на основе экспертной оценки сотрудниками и руководителями компаний с помощью web-сервиса «Поиск имплицитных параметров».

Для этого руководство и сотрудники компании по интервальной шкале «очень слабо», «слабо», «средне», «сильно», «очень сильно» оценили взаимное влияние показателей (рис. 3).

Полученные результаты были импортированы в Excel и отфильтрованы таким образом, чтобы идентифицировать результаты, позволяющие определить имплицитные показатели. К этим записям необходимо отнести экспертные оценки, имеющие метку «слабо» и «очень слабо», которым соответствуют модельные оценки «сильно» и «очень сильно». Из результатов мы видим, в частности, что «Прибыль» слабо связано с показателем «Количество клиентов/проектов», но имеет сильное опосредованное влияние через «Затраты на привлечение одного клиента». Показатель «Затраты на привлечение одного клиента» в данном случае, согласно логике исследования, является имплицитным (рис. 4). Аналогично выявляются другие имплицитные показатели. Следует заметить, что влияние имплицитных факторов на достижение стратегической цели компании не было идентифицировано ни одним из экспертов.

На основании процедуры рефлексивного отбора было составлено каузальное поле и показатели распределены по трем группам: «Показатели имплицитного фактора», «Опосредованные показатели» и «Ключевые показатели деятельности». Экспертное распределение представлено в виде табл. 2.

Таким образом, в ходе проведенного эксперимента были отобраны показатели деятельности исследуемой организации, проведено уточнение их набора с помощью модели рефлексивного отбора, построено их каузальное поле. За счет использования интеллектуального помощника - разработанного web-сервиса количество показателей было сокращено до 13, практически на 40 \%, т. е. эмпирически доказано, что 8 показателей деятельности слабо влияют на достижение 
Прибыль влияет на
Прибыль

Прибыль влияет на Уровень издержек

Прибыль влияет на Количество клиентов/ проектов Прибыль влияет на Оценка
клиента

Прибыль влияет на Индекс удовлетворенности

Прибыль влияет на Количество привлеченных клиентов

Прибыль влияет на Затраты на привлечение одного клиента

\section{Прибыль влияет на Суммарное количество просмотров сайтов}

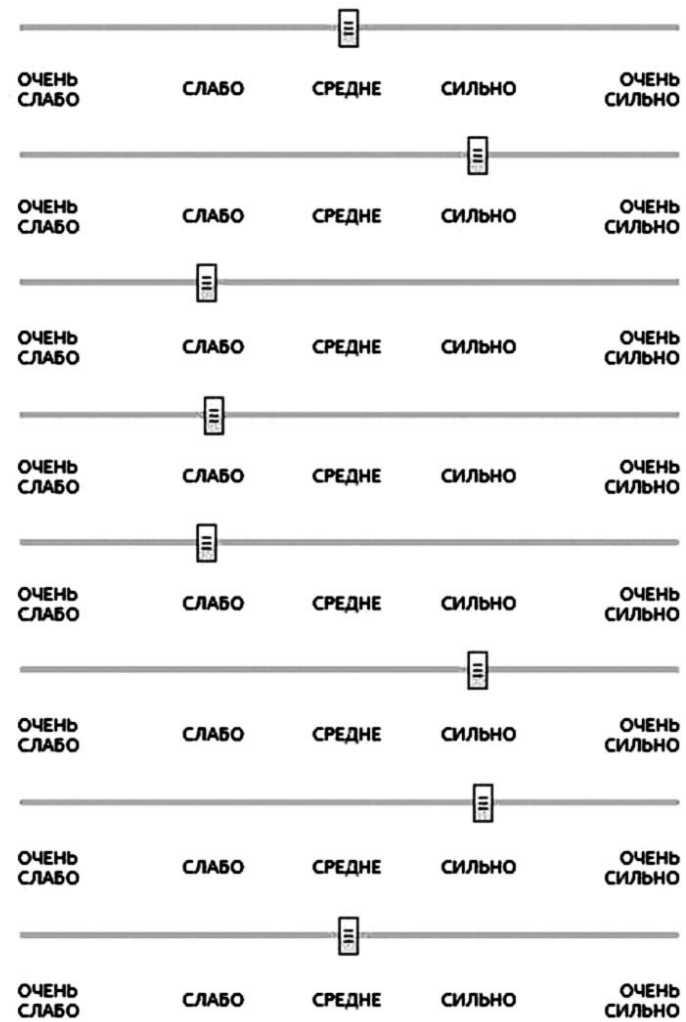

Puc. 3. Настройка влияния параметров в форме сервиса «Поиск имплицитных параметров»

\begin{tabular}{|c|c|c|c|}
\hline $\begin{array}{c}\text { Параметр } \\
\text { модели }\end{array}$ & $\begin{array}{c}\text { Имплицитный параметр } \\
\text { Прибыль }\end{array}$ & $\begin{array}{c}\text { Патраты на привлечение } \\
\text { одного }\end{array}$ & $\begin{array}{c}\text { Пкспертная } \\
\text { оценка }\end{array}$ \\
\hline Прибыль & $\begin{array}{c}\text { Затраты на привлечение } \\
\text { одного }\end{array}$ & $\begin{array}{c}\text { Количество } \\
\text { клиентов/проектов }\end{array}$ & Слабо \\
\hline Прибыль & $\begin{array}{c}\text { Количество привлеченных } \\
\text { клиентов }\end{array}$ & Оценка клиента & Слабо \\
\hline Прибыль & $\begin{array}{c}\text { Количество привлеченных } \\
\text { клиентов }\end{array}$ & $\begin{array}{c}\text { Индекс } \\
\text { удовлетворенности }\end{array}$ & Слабо \\
\hline Прибыль & $\begin{array}{c}\text { Количество привлеченных } \\
\text { клиентов }\end{array}$ & $\begin{array}{c}\text { Суммарное } \\
\text { количество } \\
\text { просмотров }\end{array}$ & Слабо \\
\hline Уровень издержек & $\begin{array}{c}\text { Количество привлеченных } \\
\text { клиентов }\end{array}$ & $\begin{array}{c}\text { Прибыль } \\
\text { клиентов }\end{array}$ & Очень слабо \\
\hline Уровень издержек & $\begin{array}{c}\text { Количество привлеченных } \\
\text { Оценка клиента }\end{array}$ & Очень слабо \\
\hline кровень издержек & $\begin{array}{c}\text { Количество привлеченных } \\
\text { клиентов }\end{array}$ & $\begin{array}{c}\text { Индекс } \\
\text { удовлетворенности }\end{array}$ & Слабо \\
\hline Уровень издержек & $\begin{array}{c}\text { Затраты на } \\
\text { Прибыль }\end{array}$ & $\begin{array}{c}\text { привлечение одного } \\
\text { клиена }\end{array}$ & Слабо \\
\hline
\end{tabular}

Рuc. 4. Фрагмент промежуточных результатов выявления имплицитных показателей 
стратегических целей компании и их мониторинг повлечет неоправданные издержки (которые также представляют собой имплицитный фактор в системе управления организацией, оказывающий влияние на достижение стратегической цели, выраженной показателем «снижение издержек»).

\section{Таблица 2. Система показателей деятельности ООО «ИТЕРУС», распределенных по трем исследуемым подмоделям}

\begin{tabular}{|c|c|c|}
\hline $\begin{array}{c}\text { Показатели корпоративной культуры } \\
\text { (имплицитный фактор) } \\
\text { (Подмодель } A \text { ) }\end{array}$ & $\begin{array}{c}\text { Опосредованные } \\
\text { (промежуточные) } \\
\text { показатели (Подмодель } B \text { ) }\end{array}$ & $\begin{array}{c}\text { Основные показатели } \\
\text { деятельности компании } \\
\text { (Подмодель } C)\end{array}$ \\
\hline $\begin{array}{l}\text { Количество привлеченных клиентов } \\
\text { Суммарное количество просмотров } \\
\text { сайтов и групп компании } \\
\text { Уровень производительности труда } \\
\text { Проекты без просрочки (доля в общем } \\
\text { кол-ве) } \\
\text { Индекс удовлетворенности сотрудников } \\
\text { Кол-вообщих собраний } \\
\text { Доля уволившихся сотрудников в общей } \\
\text { численности персонала } \\
\text { Затраты на привлечение клиента }\end{array}$ & $\begin{array}{l}\text { Оценка клиента } \\
\text { Индекс удовлетворенности } \\
\text { Позиция сайта }\end{array}$ & $\begin{array}{l}\text { Прибыль } \\
\text { Уровень издержек }\end{array}$ \\
\hline
\end{tabular}

\section{Выводы}

Проблема выявления и учета имплицитных факторов, а также оценки их влияний на эффективность деятельности организации многоаспектна и требует серьезных научных исследований на фоне их возрастающего количества и увеличивающейся значимости в процессе принятия решений в условиях информационной экономики. Не менее остро стоит проблема толкования имплицитных факторов в процессе управления организацией с целью прогнозирования сценариев развития ее деятельности. Решение обозначенных проблем позволит повысить эффективность управления организацией, а в конечном счете - улучшить экономическое положение организации на соответствующем сегменте рынка с минимальными издержками. Вклад настоящего исследования заключается в разработке интеллектуального обеспечения рефлексивного управления в ССП, результатом которой является модель определения имплицитных факторов в системе сбалансированных показателей деятельности организации, реализованная в виде web-сервиса «Поиск имплицитных параметров». Особенности модели, реализующей функцию рефлексивного отбора в рамках ССП, заключаются в следующем.

1. Выделение показателей, характеризующих имплицитные факторы в системе ценностей ЛПР рассматриваемой организации.

2. Показатели имплицитных факторов (подмодель А) влияют на ключевые показатели деятельности организации (подмодель С) не напрямую, а опосредованно, через систему измеряемых показателей подмодели В, связанных, с одной стороны, с основными, с другой - с имплицитными факторами.

3. Главным действующим лицом, осуществляющим окончательный выбор тех или иных показателей (по формуле Лефевра: «истина» - «ложь») в рамках процедуры рефлексивного отбора, являются руководитель и владельцы основных биз- 
нес-процессов, которые действуют на основе своего опыта в рамках корпоративной системы ценностей, а также с использованием систем интеллектуального анализа данных, аккумулирующих знания организации.

4. Возможность согласовать практически все изменения показателей, заложенных в стратегической карте, и, таким образом, преобразовать желаемые руководством оценки в измеримые параметры, применяя сервис «Оценка влияния имплицитных факторов на основные показатели деятельности организации».

\section{Литература}

Галахов И.В. Проектирование корпоративной информационно-аналитической системы. URL: http:// www.osp.ru/text/302/182903/12.03.2013 (дата обращения: 17.01. 2016).

Каплан Р. С., Нортон Д. П. Сбалансированная система показателей: от стратегии к действию. 2-е изд., испр. и доп. / пер. с англ. М.: ЗАО «Олимп-Бизнес», 2005. 304 с.

Канке В. А. Философия экономической науки: учеб. пособие. М.: ИНФРА-М, 2009. 384 с.

Лефевр В.А., Смолян Г.Л. Алгебра конфликта. М.: Знание, 1968. 50 с.

Лефевр В. А. Лекции по теории рефлексивных игр. М.: Когито-Центр, 2009. 218 с.

Лепский В.Е. Рефлексивно-активные среды инновационного развития. URL: http://gtmarket.ru/laboratory/expertize/6728/6734 (дата обращения: 20.03. 2017).

Конышева Л.К., Назаров Д.М. Основы теории нечетких множеств: учеб. пособие. СПб.: Питер, 2011. $192 \mathrm{c}$.

Назаров Д. М. Методология нечетко-множественной оценки имплицитных факторов в деятельности организации. Екатеринбург: Изд-во Урал. гос. экон. ун-та, 2016. 193 с.

Назаров Д. М., Давыљкин Е. В. Синергетическая сущность управления корпоративной культурой труда как бизнес-процессом промышленного предприятия // Экономический анализ: теория и практика. 2012. № 37. С. 15-20.

Недосекин А. О., Шкатов М. Ю., Абдулаева 3. И. Разработка системы сбалансированных показателей для морской нефтегазовой смешанной компании с использованием нечетко-множественных описаний // Аудит и финансовый анализ. 2013. № 4. С. 126-134.

Нейман Дж. фон, Моргенштерн О. Теория игр и экономическое поведение. М.: Наука, 1970. 708 с.

Ольве Н.-Г., Рой Ж., Веттер М. Оценка эффективности деятельности компании: практическое руководство по использованию сбалансированной системы показателей / пер. с англ. М.: Издательский дом «Вильямс», 2004. 304 с.

Лепа Л.Н., Мальчик М.В. Рефлексивное управление конкурентоспособностью промышленных предприятий. Донецк-Ровно: ЧП Лапсюк В. А., 2010. 304 с.

Экономическая теория на пороге XXI века / под ред. Ю. М. Осипова, В. Т. Пуляева, В. Т. Рязанова, Е. С. Зотовой. М.: Юристъ, 1998. 768 с.

Kahneman D., Tversky A. Prospect Theory: an Analysis of Decisions under Risk // Econoraetrica. 1979. N 47. P. 263-291.

Nazarov D. Fuzzy model for working capital speed control as an implicit factor of economy $/ / 19^{\text {th }}$ IEEE International Conference on Soft Computing and Measurements, SCM 2016. SPb: Saint Petersburg Electrotechnical University (SPbETU), 2016. P. 487-488.

North D. C. Institutions // Journal of Economic Perspectives. 1991. Vol. 5, N 1. P.97-112.

Fogel R. W. Railroads and American Economic Growth: Essays in Econometric History. Baltimore: Johns Hopkins Press, 1964. xv +296 pp.

George J. Stigler, Textual Exegesis as a Scientific Problem // Economica. New Series. 1965. Vol. 32, N 128. P. 447450.

Для цитирования: Назаров Д.М. Модель рефлексивного отбора имплицитных показателей управленческой деятельности организации // Вестник СПбГУ. Экономика. 2017. Т.33. Вып. 3. С. 498518. DOI: $10.21638 / 11701 /$ spbu05.2017.308.

\section{References}

Ekonomicheskaia teoriia na poroge XXI veka [Economic theory on the threshold of the XXI century]. Eds. Iu. M. Osipov, V.T. Puliaev, V.T. Riazanov, E. S. Zotova. Moscow, Iurist" Publ., 1998. 768 p. (In Russian) 
Fogel R. W. Railroads and American Economic Growth: Essays in Econometric History. Baltimore, Johns Hopkins Press, 1964. xv + $296 \mathrm{p}$.

Galakhov I.V. Proektirovanie korporativnoi informatsionno-analiticheskoi sistemy [Designing a corporate information and analytical system]. Available at: http://www.osp.ru/text/302/182903/12.03.2013 (accessed: 17.01. 2016). (In Russian)

George J. Stigler, Textual Exegesis as a Scientific Problem. Economica. New Series, 1965, vol. 32, no. 128, pp. 447450 .

Kahneman D., Tversky A. Prospect Theory: an Analysis of Decisions under Risk. Econoraetrica, 1979, no. 47, pp. 263-291.

Kanke V. A. Filosofiia ekonomicheskoi nauki: ucheb. posobie [Philosophy of Economic Science: Proc. Allowance]. Moscow, INFRA-M Publ., 2009. 384 p. (In Russian)

Kaplan R. S., Norton D. P. Sbalansirovannaia sistema pokazatelei: ot strategii k deistviiu [Balanced scorecard: from strategy to action]. $2^{\text {nd }}$ ed., ispr. i dop. Transl. from English. Moscow, ZAO “Olimp-Biznes” Publ., 2005. 304 p. (In Russian)

Konysheva L. K., Nazarov D. M. Osnovy teorii nechetkikh mnozhestv: ucheb. posobie [Fundamentals of the theory of fuzzy sets: training aids]. St. Petersburg, Piter Publ., 2011. 192 p. (In Russian)

Lefevr V. A. Lektsii po teorii refleksivnykh igr [Lectures on the theory of reflexive games]. Moscow, Kogito-Tsentr Publ., 2009. 218 p. (In Russian)

Lefevr V.A., Smolian G. L. Algebra konflikta [The Algebra of Conflict]. Moscow, Znanie Publ., 1968.50 p. (In Russian)

Lepa L.N., Mal'chik M.V. Refleksivnoe upravlenie konkurentosposobnost'iu promyshlennykh predpriiatii [Reflexive management of competitiveness of industrial enterprises]. Donetsk, Rovno, ChP Lapsiuk V.A., 2010. 304 p. (In Russian)

Lepskii V.E. Refleksivno-aktivnye sredy innovatsionnogo razvitiia [Reflexively active mediums of innovative development]. Available at: http://gtmarket.ru/laboratory/expertize/6728/6734 (accessed: 20.03. 2017).

Nazarov D. Fuzzy model for working capital speed control as an implicit factor of economy. $19^{\text {th }}$ IEEE International Conference on Soft Computing and Measure-ments, SCM 2016. St. Petersburg, Saint Petersburg Electrotechnical University Publ., 2016, pp. 487-488.

Nazarov D. M. Metodologiia nechetko-mnozhestvennoi otsenki implitsitnykh fak-torov $v$ deiatel'nosti organizatsii [Methodology of fuzzy-multiple estimation of implicit factors in the organization's ac-tivities]. Ekaterinburg, Publ. Ural. gos. ekon. un-ta, 2016. 193 p. (In Russian)

Nazarov D. M., Davydkin E. V. Sinergeticheskaia sushchnost' upravleniia korpora-tivnoi kul'turoi truda kak biznes-protsessom promyshlennogo predpriiatiia [Synergetic essence of the management of corporate culture of labor as a business process of an industrial enterprise]. Ekonomicheskii analiz: teoriia i praktika [Economic analysis: theory and practice], 2012, no. 37, pp. 15-20. (In Russian)

Nedosekin A.O., Shkatov M.Iu., Abdulaeva Z.I. Razrabotka sistemy sbalansi-rovannykh pokazatelei dlia morskoi neftegazovoi smeshannoi kompanii s is-pol'zovaniem nechetko-mnozhestvennykh opisanii [Development of a system of balanced indicators for a marine oil and gas mixed com-pany using fuzzy descriptions]. Audit i finansovyi analiz [Audit and financial analysis], 2013, no. 4, pp. 126-134. (In Russian)

Neiman Dzh. fon, Morgenshtern O. Teoriia igr i ekonomicheskoe povedenie [Game theory and economic behavior]. Moscow, Nauka Publ., 1970. 708 p. (In Russian)

North D. C. Institutions. Journal of Economic Perspectives, 1991, vol. 5, no. 1, pp.97-112.

Ol've N.-G., Roi Zh., Vetter M. Otsenka effektivnosti deiatel'nosti kompa-nii: prakticheskoe rukovodstvo po ispol'zovaniiu sbalansirovannoi sistemy pokazatelei [Evaluation of the effectiveness of the company: a practical guide to the use of a bal-anced system of indicators]. Transl. from Engl. Moscow, Publ. Vil'iamc, 2004. 304 p. (In Russian)

For citation: Nazarov D. M. Model of reflexive selection of implicit indicators of management activities of an organization. St Petersburg University Journal of Economic Studies, 2017, vol.33, issue 3, pp. 498-518. DOI: $10.21638 / 11701 /$ spbu05.2017.308. 